\title{
Commentary on the Presence of Periodontal Pathogens in Gastric Cancer
}

\author{
Adamu Danbala Isah ${ }^{1}$, Xiaofei Xue ${ }^{2}$, Xiaogang Wang ${ }^{2}$ and Shengchun Dang ${ }^{1,2 *}$ \\ ${ }^{1}$ Department of Gastrointestinal Surgery, Affiliated Hospital of Jiangsu University, Zhenjiang, Jiangsu, China; \\ ${ }^{2}$ Department of General Surgery, Pucheng Hospital, Weinan, Shaanxi, China
}

\begin{abstract}
A recent study published by Marcel et al., ${ }^{1}$ titled "The Presence of Periodontal Pathogens in Gastric Cancer" in Explor Res Hypothesis Med draws our attention. The human microbiome (HM) is the collection of all microbiota that are found on or within human body tissues, along with the communicating anatomical sites in which they are found. They mainly present in the gastrointestinal tract, placenta, skin, oronasal mucosa, biliary tract, uterus, and seminal fluid. The HM also indicates the mutual genomes of the encompassed microbes, which generally consist of viruses, bacteria, and protozoa that are found in the human body. ${ }^{2}$ The oral microflora comprises several ecological biofilms that are colonized mainly from birth through life and up to death. ${ }^{3}$ They may play an important role in the pathogenesis of some diseases. However, few works have been focused on the precise position and formation of oral microflora.
\end{abstract}

In their research, Wu et al., ${ }^{4}$ have evaluated the precise location (position) and the formation of oral microflora in many oral types of infections. The majority of these infections were caused by viruses and bacteria. The diseases they studied are considered as chronic diseases, such as cancer and diabetes mellitus. Their findings prompt the possibility of involving various types of microbes in the development and progression of other chronic diseases.

Several strengths of this study are noteworthy. First, it helps better understand the presence of periodontal pathogens in gastric cancer (GC). Second, the analytical method of the amplicon sequence variant (ASV) was rightfully used in their study. Third, the authors found a considerable number of opportunistic pathogens, of which the majority are known periodontal pathogens and not yet considered as carcinogenic. ${ }^{1}$ Fourth, the inclusion of healthy subjects in the study also significantly increased the scientific rigor of their work. Finally, the study also identified Helicobacter pylori as a risk factor for $\mathrm{GC}$, which has been confirmed as a causative organism of GC.

However, evaluation of the previous studies of about a hundred subjects indicated that, among the individuals with significant gastric H. pylori, at most, $44.9 \%$ did not exhibit the prevalence of $H$. pylori mainly in saliva and $42.9 \%$ exhibited $H$. pylori certainly in the supra-gingival plate. ${ }^{5}$ Among the patients and individuals who did not show gastric $H$. pylori, $53.1 \%$ manifested the exist-

Abbreviations: ASV, amplicon sequence variant; GC, gastric cancer; HM, human microbiome.

Received: June 21, 2020; Revised: June 24, 2020; Accepted: June 30, 2020

*Correspondence to: Shengchun Dang, Department of Gastrointestinal Surgery, Affiliated Hospital of Jiangsu University, Zhenjiang 212000, Jiangsu, China. Tel: +86511-85082208; Fax: +86-511-88820988; E-mail: dscgu@163.com

How to cite this article: Isah $\mathrm{AD}$, Xue $\mathrm{X}$, Wang $\mathrm{X}$, Dang S. Commentary on the Presence of Periodontal Pathogens in Gastric Cancer. Exploratory Research and Hypothesis in Medicine 2020;5(3):75-76. doi: 10.14218/ERHM.2020.00043. ence of infection mainly in saliva and almost $42.8 \%$ in supra-gingival plates. ${ }^{6}$ Besides, the presence of Fusobacterium nucleatum, a known colorectal cancer-associated pathogen, among the top four pathogens is also interesting. The authors also found 17 distinct ASVs assigned to Propionibacterium acnes and 53 distinct ASVs allocated to $F$. nucleatum in this dataset. ${ }^{1}$

This study is novel in its investigation on both $H$. pylori and $P$. acne together, because most studies have not performed such analysis. Although, $P$. acne is a significant pathogen affecting human health, causing a variety of postoperative and device-associated infections. It mainly colonizes the deep hair follicles and is commonly found on the skin of even healthy persons. P. acnes' initial sources of nutrients are the cellular debris from the proximal skin tissue. Hence, it is not surprising that this study found the prevalence of the skin pathogen P. acnes (at $60 \%$ ). ${ }^{1}$ F. nucleatum was of our particular interest. For instance, how the Gram-negative bacterium $F$. nucleatum initiates the overall tumor progression by comprising inflammation as well as host immunity mainly in the colorectal cancer niche? Furthermore, F. nucleatum is known as a constituent of the oral plate, while it is also known as an initial and final colonizer of biofilm. How the oral plate component contributes to the carcinogenesis of GC is intriguing and, of course, important. Besides, F. nucleatum is a type of highly diverse species and has been difficult to classify or categorize. Currently, some subspecies are illustrated, such as nucleatum, animals, vincentii, and polymorphism.

In summary, this study is important despite its limitations. At the time of treatment, the authors show that probiotics should be accepted as a treatment choice, after $H$. pylori eradication therapy, to avoid recolonization by periodontal pathogens. ${ }^{1}$ Moreover, Rothen $^{7}$ determined that establishing proper oral hygiene (e.g., tooth brushing at least twice a day) can be useful in limiting the development of periodontal diseases or the spread of cancer. Finally, the three species identified by the ecological network analysis also represent the tumor-associated species that overlap between four $\mathrm{GC}$ and colorectal cancer datasets as shown by the researchers. ${ }^{1}$

\section{Acknowledgments}

The authors thank Qais Ahmad Naseer for linguistic assistance during the preparation of this manuscript.

\section{Funding}

This work was supported by the Zhenjiang Science and Technology Project (SH2019061). 
Explor Res Hypothesis Med

\section{Conflict of interest}

The authors have no conflicts of interest related to this publication.

\section{Author contributions}

Review of the literature and drafting of the manuscript (ADI, XXF, and $\mathrm{WXG}$ ), critical revision of the manuscript (DSC).

\section{References}

[1] de Leeuw MA, Duval MX. The Presence of Periodontal Pathogens in Gastric Cancer. Explor Res Hypothesis Med 2020. doi:10.14218/ ERHM.2020.00024.

[2] Gholizadeh P, Eslami H, Yousefi M, Asgharzadeh M, Aghazadeh M, Kafil HS. Role of oral microbiome on oral cancers, a review. Biomed
Isah AD. et al: Commentary on periodontal pathogens in GC

Pharmacother 2016;84:552-558. doi:10.1016/j.biopha.2016.09.082.

[3] Bhushan B, Singh BP, Kumari M, Mishra V, Saini K, Singh D. Microbes, their metabolites, and effector molecules: a pharmacological perspective for host-microbiota interaction. In: Arora D, Sharma C, Jaglan S, Lichtfouse E. (eds) Pharmaceuticals from Microbes, Vol. 28. Springer. 2019:155-206. doi:10.1007/978-3-030-04675-0_7.

[4] Wu J, Xu S, Xiang C, Cao Q, Li Q, Huang J, et al. Tongue Coating Microbiota Community and Risk Effect on Gastric Cancer. J Cancer 2018;9(21):4039-4048. doi:10.7150/jca.25280.

[5] Yao J, Kovalik JP, Lai OF, Lee PC, Eng A, Chan WH, et al. Comprehensive assessment of the effects of sleeve gastrectomy on glucose, lipid, and amino acid metabolism in asian individuals with morbid obesity. Obes Surg 2019;29(1):149-158. doi:10.1007/s11695-0183487-2.

[6] Genco RJ, Sanz M. Clinical and public health implications of periodontal and systemic diseases: An overview. Periodontol 2000 2020;83(1):7-13. doi:10.1111/prd.12344.

[7] Rothen ML. Oral Hygiene and Prevention for CSHCN. In: Nelson T, Webb J. (eds) Dental Care for Children with Special Needs, Springer. 2019:141-166. doi:10.1007/978-3-030-10483-2_7. 